\title{
BAYESIAN MODEL AVERAGING IN CONSUMER DEMAND SYSTEMS WITH INEQUALITY CONSTRAINTS
}

\author{
C.L Chua \\ mchua@unimelb.edu.au \\ Melbourne Institute of Applied Economic and Social Research \\ University of Melbourne 3010 \\ Australia
}

W.E. Griffiths

b.griffiths@unimelb.edu.au

Department of Economics

University of Melbourne 3010

Australia

\section{C.J O'Donnell}

codonnel@metz.une.edu.au

School of Economics

University of New England

Armidale 2351, Australia

http://www.ecom.unimelb.edu.au/ecowww/wpapers.html

Paper prepared for Frontier Session on Markov Chain Monte Carlo Methods, American Agricultural Economics Association Meetings, August 5-7, 2001, Chicago. 


\begin{abstract}
Share equations for the translog and almost ideal demand systems are estimated using Markov Chain Monte Carlo. A common prior on the elasticities and budget shares evaluated at average prices and income is used for both models. It includes equality restrictions (homogeneity, adding up and symmetry) and inequality restrictions (monotonicity and concavity). Posterior densities on the elasticities and shares are obtained; the problem of choosing between the results from the two alternative functional forms is resolved by using Bayesian model averaging. The application is to USDA data for beef, pork and poultry. Estimation of elasticities and shares, evaluated at mean prices and expenditure, is insensitive to model choice. At points away from the means the estimates are sensitive, and model averaging has an impact.

JEL classifications: C11, C32, E21.
\end{abstract}

$\underline{\text { Keywords: }}$ conditional prior, Marginal likelihood, Metropolis-Hastings algorithm. 


\section{Introduction}

The estimation of demand systems in the form of share equations derived from flexible indirect cost or utility functions is common place. Popular examples are the the almost ideal demand system (Deaton and Muellbauer), and the translog demand system (Jorgenson, Lau and Stoker). A comprehensive review of these and other systems appears in Pollak and Wales. The main attractiveness of a flexible functional form is that the demand model has enough free parameters to provide a second order approximation to an arbitrary twice differentiable function at a particular set of prices. However, this flexibility comes at a cost. Inequality constraints on the parameters implied by theory, namely, monotonicity and concavity, are not automatically satisfied. Consequently, methods for imposing these constraints have been investigated. Examples within a sampling theory framework are Ryan and Wales, and Moschini, and, within a Bayesian framework, Chalfant, Gray and White, and Gordon. Using a Bayesian framework to investigate this question further is one of the main objectives of this paper. In addition, we address the question of Bayesian model averaging of alternative functional forms. Traditionally, choice of functional form has been based on goodness-of-fit criteria or on a series of nested or nonnested hypothesis tests designed to discriminate between alternative models. One problem with this practice is that, once a particular model has been chosen, the fact that a number of other models have been discarded is usually ignored. No allowance is made for the possibility of sample statistics yielding an incorrect choice. Assessment of the precision of estimation via standard errors makes no provision for the preliminary-test implications for inference. The preliminary-test problem has received considerable attention in the sampling-theory literature (see, for example, Judge and Bock, and 
Danilov and Magnus), but most of the solutions are for particular special cases and do not carry over to model selection problems like the one considered here.

Bayesian model averaging along the lines described by Geweke provides an alternative that does yield results and measures of precision that reflect model uncertainty. In this approach the results from each model are combined, as a weighted average, with the weight attached to each model being the posterior probability that that model is "correct". If one model is vastly superior to the others, then its posterior probability will be close to one, and the averaged results will not be distinguishable from those of the best-fitting superior model. On the other hand, if the choice between models is a less definite one, then each of the models will contribute information to the averaged-results, and measures of precision, such as posterior standard deviations, will reflect the model uncertainty.

To illustrate the Bayesian modeling averaging procedure in the context of demand systems with inequality constraints, we estimate two nonnested systems, an almost ideal demand system (AIDS) and a log-translog system (LTL). Both are applied to quarterly data on U.S. beef, pork and poultry consumption for the period 1979(1) to 1995(2). These data were kindly provided by Nick Piggott who used them within another context (Piggott; Alston, Chalfant and Piggott). The procedure we follow is depicted in Figure 1. We begin by choosing some economic quantities of interest (EQI) denoted by the vector $\theta$; a prior probability density function (pdf), $p(\theta)$, is then assigned to these EQI. Since the parameter vectors for the two models (denoted by $\gamma_{M_{1}}$ and $\gamma_{M_{2}}$ ) have different meanings, we need some kind of device for ensuring that their prior pdf's, $p\left(\gamma_{M_{1}}\right)$ and $p\left(\gamma_{M_{2}}\right)$, convey similar prior information. Beginning with a common prior on $\theta$ and transforming it to $p\left(\gamma_{M_{1}}\right)$ and $p\left(\gamma_{M_{2}}\right)$ is 
one way of achieving this objective. The EQI we chose are the expenditure shares for each of the meat categories, and all direct and cross price elasticities, and expenditure elasticities, evaluated at mean prices and mean total expenditure. The imposition of Slutsky symmetry conditions, homogeneity and adding up means that some of the EQI (and some of the model parameters) will be redundant. Modification of the prior $p(\theta)$ is required to accommodate this redundancy.

After specifying the prior $p(\theta)$ and transforming it to the priors $p\left(\gamma_{M_{1}}\right)$ and $p\left(\gamma_{M_{2}}\right)$, Bayes' theorem is used to combine these priors with likelihood functions $p\left(y \mid \gamma_{M_{i}}, M_{i}\right), i=1,2$, to obtain the posterior pdf's $p\left(\gamma_{M_{i}} \mid y, M_{i}\right)$, for the parameters of models $M_{1}$ and $M_{2}$. In these expressions we use the symbol $y$ generically, to denote the sample data. Also, in practice, the complete prior and posterior pdf's will involve the unknown error covariance matrix; we have abstracted from this fact in Figure 1. Because the models $M_{1}$ and $M_{2}$ are both systems of nonlinear seemingly unrelated regressions, with inequality constraints (for concavity and monotonicity) on the parameters, the joint posterior pdf's are not analytically tractable; expressions for marginal posterior pdf's for single parameters, and their moments, cannot be obtained. A Metropolis - Hastings algorithm is used to draw observations from the joint posterior pdf's for each of the model's parameters. These observations are used to estimate marginal posterior pdf's and their moments for single elements in the vectors $\gamma_{M_{1}}$ and $\gamma_{M_{2}}$. Perhaps more importantly, for each of the posterior draws of $\gamma_{M_{1}}$ and $\gamma_{M_{2}}$, we can compute values of the EQI. These values represent draws from the posterior pdf's for the EQI, conditional on each of the models, $p\left(\theta \mid y, M_{i}\right), i=1,2$. Posterior pdf's, means and standard deviations for each of the shares and elasticities, conditional on each of the models, are obtained from these draws. 
To obtain unconditional posterior quantities, we proceed with model averaging. The first step in this direction is to obtain the marginal likelihoods $p\left(y \mid M_{i}\right), i=1,2$. A complication that arises in the estimation of the marginal likelihoods is the need to compute estimates of the normalizing constants for the priors for each of the models. Imposition of inequality constraints at each data point in the sample truncates the prior for each model in a way that is dependent on the model parameters. Thus, the normalizing constants will not be the same for each model and need to be estimated. We pursue this problem with a Metropolis - Hastings algorithm applied to each of the prior pdf's. Finally, combining the marginal likelihood values with prior model probabilities yields posterior model probabilities that are used to average the conditional posterior pdf's $p\left(\theta \mid y, M_{i}\right), i=1,2$, yielding the unconditional posterior pdf for the EQI, $p(\theta \mid y)$. In practice, we use functions of the draws of $\theta$ from the conditional posterior pdf's and average those to estimate corresponding unconditional posterior pdf's of those functions.

For the data set that we employ, we find that posterior information about the shares and elasticities, evaluated at mean prices and expenditures, is insensitive to choice between the AIDS and LTL models. Consequently, the Bayesian modelaveraged results at these points are not distinguishable from those conditional on either of the two models. However, for some points evaluated away from the means, the two models lead to different posterior information, and the averaging process has an impact on our inferences.

The framework of the paper is as follows. In the next section we describe the AIDS and LTL models, giving expressions for the EQI, and details of the equality and inequality constraints. The likelihood function is specified. The prior specification and 
modification of it to accommodate the equality restrictions are the subject of the third section. The next section contains the posterior pdf's and results for the model parameters. Model averaging is described in the fifth section. Posterior results for the EQI, including those from model averaging, are discussed in section six. Some concluding remarks are made in the final section.

\section{Models}

In this section we describe the two models (AIDS and LTL) that are averaged in the paper, the equality and inequality constraints on their parameters, and the likelihood functions.

\section{Almost Ideal Demand System}

The budget shares equations for the AIDS model take the form

$$
s_{i}=\alpha_{A i}+\sum_{j=1}^{3} \gamma_{A i j} \log p_{j}+\beta_{A i}(\log x-\log g(p)) \quad i=1,2,3
$$

where $\left(s_{1}, s_{2}, s_{3}\right)$ are the expenditure shares for beef, pork and poultry, relative to total expenditure on these three commodities. Their corresponding prices are $\left(p_{1}, p_{2}, p_{3}\right)$, $\log g(p)=\alpha_{A 0}+\sum_{k=1}^{3} \alpha_{A k} \log p_{k}+\frac{1}{2} \sum_{k=1}^{3} \sum_{j=1}^{3} \gamma_{A k j} \log p_{k} \log p_{j}$, and $x$ is total expenditure.

The unknown parameters are $\left(\left(\alpha_{A 0}, \alpha_{A i}, \beta_{A i}, \gamma_{A i j}\right), i, j=1,2,3\right)$, with the subscript $A$ being used to denote the AIDS model. Estimation of the parameter $\alpha_{A 0}$ is often problematic, yielding flat regions in the likelihood surface; we set $\alpha_{A 0}=0$, implying a zero minimum outlay on each commodity. To ensure the theoretical properties of adding up, homogeneity and symmetry are satisfied, the following equality constraints are imposed on the parameters: 


$$
\sum_{i=1}^{3} \alpha_{A i}=1, \quad \sum_{i=1}^{3} \beta_{A i}=0, \quad \sum_{j=1}^{3} \gamma_{A i j}=0, \quad \gamma_{A k j}=\gamma_{A j k}
$$

The price elasticity of demand for commodity $i$ with respect to price $j$ is

$$
\eta_{i j}=\frac{\gamma_{A i j}}{s_{i}}-\delta_{i j}-\frac{\beta_{A i}}{s_{i}}\left(\alpha_{A j}+\sum_{k=1}^{3} \gamma_{A j k} \log p_{k}\right)
$$

where $\delta_{i j}=1$ for $i=j$, and 0 otherwise. The expenditure elasticity for commodity $i$ is

$$
\eta_{i x}=\frac{\beta_{A i}}{s_{i}}+1
$$

For monotonicity to hold we require $0<s_{i}<1, i=1,2,3$. For concavity to hold the socalled Slutsky matrix must be negative semidefinite. Given monoticity holds, this matrix will be negative semidefinite if and only if the $(3 \times 3)$ matrix with $(i, j)$ th element $s_{i} \eta_{i j}+s_{i} s_{j} \eta_{i x}$ is negative semidefinite. Clearly, the shares and elasticities depend on the prices, total expenditure and the parameter values. In our empirical work the shares were evaluated as predictions from equation (1), and monotonicity and concavity were imposed locally at every data point in the sample. Negative semidefiniteness of the Slutsky matrix was ensured by constraining the largest eigenvalue to be nonpositive.

\section{Log Translog Demand System}

The expenditure share equations for the LTL system are given by

$$
s_{i}=\frac{\alpha_{B i}+\sum_{j=1}^{3} \gamma_{B i j} \log p_{j}-\log x \sum_{j=1}^{3} \gamma_{B i j}}{1+\sum_{k=1}^{3} \sum_{j=1}^{3} \gamma_{B k j} \log p_{j}} \quad i=1,2,3
$$


The variable definitions carry over from the AIDS model. The parameters have been subscripted with $B$ to denote the LTL model. Homogeneity is always satisfied. To ensure adding up and symmetry we impose the equality constraints

$$
\sum_{i=1}^{3} \alpha_{B i}=1 \quad \sum_{i=1}^{3} \sum_{j=1}^{3} \gamma_{B i j}=0 \quad \gamma_{B k j}=\gamma_{B j k}
$$

Let $D=1+\sum_{k=1}^{3} \sum_{j=1}^{3} \gamma_{B k j} \log p_{j}$. The price elasticity of demand for commodity $i$ with respect to price $j$ is

$$
\eta_{i j}=\frac{\gamma_{B i j}}{s_{i} D}-\delta_{i j}-\frac{\sum_{k=1}^{3} \gamma_{B k j}}{D}
$$

The expenditure elasticity for commodity $i$ is

$$
\eta_{i x}=-\frac{\sum_{j=1}^{3} \gamma_{B i j}}{s_{i} D}+1
$$

The inequality constraints implied by monotonicity and concavity are the same as those for the AIDS model except that the shares and elasticities are computed using equations (5), (7) and (8).

\section{The Likelihood Specification}

In both models the equality restrictions on the parameters imply that the parameters of one equation can always be deduced from those of the other two. Consequently, we can focus on estimation of two equations from the three-equation system; we chose those for beef and pork $(i=1,2)$. Also, after using the restrictions in (2) and (6) to substitute out the remaining redundant parameters, there are 7 parameters remaining in each system. We denote the vectors of these remaining 
parameters by $\gamma_{A}$ and $\gamma_{B}$. In line with the substitutions that we chose, they are given by

$$
\begin{aligned}
& \gamma_{A}=\left(\begin{array}{lllllll}
\alpha_{A 1} & \alpha_{A 2} & \gamma_{A 11} & \gamma_{A 12} & \gamma_{A 22} & \beta_{A 1} & \beta_{A 2}
\end{array}\right)^{\prime} \\
& \gamma_{B}=\left(\begin{array}{lllllll}
\alpha_{B 1} & \alpha_{B 2} & \gamma_{B 11} & \gamma_{B 12} & \gamma_{B 13} & \gamma_{B 22} & \gamma_{B 23}
\end{array}\right)^{\prime}
\end{aligned}
$$

Working towards a description of the likelihood functions, we modify the first two equations in (1) to include all $T$ observations and stochastic error terms so that they can be written as

$$
s=\left(\begin{array}{l}
s_{1} \\
s_{2}
\end{array}\right)=\left(\begin{array}{l}
f_{A 1}\left(p, x, \gamma_{A}\right) \\
f_{A 2}\left(p, x, \gamma_{A}\right)
\end{array}\right)+\left(\begin{array}{l}
\varepsilon_{1} \\
\varepsilon_{2}
\end{array}\right)=f_{A}\left(p, x, \gamma_{A}\right)+\varepsilon
$$

where $s_{i}, f_{A i}($.$) and \varepsilon_{i},(i=1,2)$, are $T$-dimensional vectors and $s, f_{A}($.$) and \varepsilon$ are $2 T$-dimensional vectors. We assume that the bivariate observations $\left(\varepsilon_{1 t}, \varepsilon_{2 t}\right)^{\prime}$, $t=1,2, \ldots, T$, are independent normally distributed random vectors with mean vector zero and covariance matrix $\Sigma_{A}$. Then, the likelihood function can be written as

$$
\begin{aligned}
p\left(s \mid \gamma_{A}, \Sigma_{A}, M_{A}\right) & \propto\left|\Sigma_{A}\right|^{-T / 2} \exp \left\{-\frac{1}{2}\left(s-f_{A}\left(p, x, \gamma_{A}\right)\right)^{\prime}\left(\Sigma_{A}^{-1} \otimes I\right)\left(s-f_{A}\left(p, x, \gamma_{A}\right)\right)\right\} \\
& =\left|\Sigma_{A}\right|^{-T / 2} \exp \left\{-\frac{1}{2} \operatorname{tr}\left(W_{A} \Sigma_{A}^{-1}\right)\right\}
\end{aligned}
$$

where the elements of $W_{A}$ are given by $w_{A i j}=\left(s_{i}-f_{A i}\left(p, x, \gamma_{A}\right)\right)^{\prime}\left(s_{j}-f_{A j}\left(p, x, \gamma_{A}\right)\right)$, $i=1,2$. The equations and likelihood are in the form of a set of nonlinear seemingly unrelated regression equations. See, for example, Judge et al (p.551).

Using analogous definitions, assumptions and notation, the likelihood function for the LTL system can be written as 


$$
\begin{aligned}
p\left(s \mid \gamma_{B}, \Sigma_{B}, M_{B}\right) & \propto\left|\Sigma_{B}\right|^{-T / 2} \exp \left\{-\frac{1}{2}\left(s-f_{B}\left(p, x, \gamma_{B}\right)\right)^{\prime}\left(\Sigma_{B}^{-1} \otimes I\right)\left(s-f_{B}\left(p, x, \gamma_{B}\right)\right)\right\} \\
& =\left|\Sigma_{B}\right|^{-T / 2} \exp \left\{-\frac{1}{2} \operatorname{tr}\left(W_{B} \Sigma_{B}^{-1}\right)\right\}
\end{aligned}
$$

\section{Prior Specification}

Before Bayes' theorem can be applied to the likelihood functions in (11) and (12), we need to specify prior pdf's $p\left(\gamma_{A}, \Sigma_{A}\right)$ and $p\left(\gamma_{B}, \Sigma_{B}\right)$. Rather than do so directly, we begin by specifying priors on the EQI (elasticities and shares), evaluated at mean prices and expenditure, so that similar prior information is conveyed for each of the models. Ignoring for the moment equality restrictions that the elasticities and shares must satisfy, we take their priors as independent and uniform, and distributed on the following intervals:
(a) $\overline{s_{1}}, \bar{s}_{2}, \bar{s}_{3} \sim U(0.05,0.95)$
(b) $\quad \bar{\eta}_{11}, \bar{\eta}_{22}, \bar{\eta}_{33} \sim U(-3,0)$
(c) $\quad \bar{\eta}_{12}, \bar{\eta}_{13}, \bar{\eta}_{21}, \bar{\eta}_{23}, \bar{\eta}_{31}, \bar{\eta}_{32} \sim U(-3,3)$
(d) $\quad \bar{\eta}_{1 x}, \bar{\eta}_{2 x}, \bar{\eta}_{3 x} \sim U(-3,3)$

The over-bar indicates quantities evaluated at mean prices and expenditure. The shares are assumed to be no less than 0.05 and no greater than 0.95. Direct price elasticities are assumed to be negative and no greater than 3 in absolute value. Crossprice and expenditure elasticities are allowed to take either sign and are also restricted to be less than 3 in absolute value. These priors were motivated by a desire to have simple, proper, and relatively noninformative priors that would be dominated by the sample information. There is nothing magical about the choice of upper and lower 
bounds; they were chosen as maximum or minimum possible values that we thought few researchers would object to.

The next step is to modify the above prior pdf to accommodate equality restrictions among the EQI. In an attempt to give a symmetric treatment to all quantities, we began with a 15-dimensional pdf. However, adding up, homogeneity and symmetry restrictions imply that 8 of the EQI are redundant. The number of nonredundant EQI is 7 , the same as the dimension of $\gamma_{A}$ and $\gamma_{B}$. To reduce the dimension of the EQI prior from 15 to 7 , in a manner consistent with the equality constraints from theory, we begin by partitioning the EQI as $\theta=\left(\theta_{1}, \theta_{2}\right)$, where

$$
\theta_{1}=\left(\begin{array}{lllllll}
\bar{\eta}_{11} & \bar{\eta}_{12} & \bar{\eta}_{13} & \bar{s}_{1} & \bar{s}_{2} & \bar{\eta}_{21} & \bar{\eta}_{32}
\end{array}\right)^{\prime}
$$

is a vector of nonredundant EQI, and the remaining EQI

$$
\theta_{2}=\left(\begin{array}{llllllll}
\bar{\eta}_{1 x} & \bar{\eta}_{2 x} & \bar{s}_{3} & \bar{\eta}_{31} & \bar{\eta}_{3 x} & \bar{\eta}_{23} & \bar{\eta}_{33} & \bar{\eta}_{22}
\end{array}\right)^{\prime}
$$

can be derived from the elements in $\theta_{1}$. The next step is to transform the prior on $\left(\theta_{1}, \theta_{2}\right)$ to one on $(\xi, \lambda)$ where $\xi=\theta_{1}$ and

$$
\lambda=\left[\begin{array}{l}
\lambda_{1} \\
\lambda_{2} \\
\lambda_{3} \\
\lambda_{4} \\
\lambda_{5} \\
\lambda_{6} \\
\lambda_{7} \\
\lambda_{8}
\end{array}\right]=\left[\begin{array}{c}
\bar{\eta}_{11}+\bar{\eta}_{12}+\bar{\eta}_{13}+\bar{\eta}_{1 x} \\
\bar{s}_{1} \bar{\eta}_{12}+\bar{s}_{1} \bar{s}_{2} \bar{\eta}_{1 x}-\bar{s}_{2} \bar{\eta}_{21}-\bar{s}_{1} \bar{s}_{2} \bar{\eta}_{2 x} \\
1-\bar{s}_{1}-\bar{s}_{2}-\bar{s}_{3} \\
\bar{s}_{1}+\bar{s}_{1} \bar{\eta}_{11}+\bar{s}_{2} \bar{\eta}_{21}+\bar{s}_{3} \bar{\eta}_{31} \\
1-\bar{s}_{1} \bar{\eta}_{1 x}-\bar{s}_{2} \bar{\eta}_{2 x}-\bar{s}_{3} \bar{\eta}_{3 x} \\
\bar{s}_{2} \bar{\eta}_{23}+\bar{s}_{2} \bar{s}_{3} \bar{\eta}_{2 x}-\bar{s}_{3} \bar{\eta}_{32}-\bar{s}_{2} \bar{s}_{3} \bar{\eta}_{3 x} \\
\bar{s}_{3}+\bar{s}_{1} \bar{\eta}_{13}+\bar{s}_{2} \bar{\eta}_{23}+\bar{s}_{3} \bar{\eta}_{33} \\
\bar{\eta}_{21}+\bar{\eta}_{22}+\bar{\eta}_{23}+\bar{\eta}_{2 x}
\end{array}\right]
$$

This transformation is chosen such that adding up, homogeneity and symmetry hold when $\lambda=0$. The partitioning of $\theta$ and consequent definition of $\lambda$ are not unique. Other alternatives could have been chosen. 
The prior on $(\xi, \lambda)$ is obtained via the transformation

$$
p(\xi, \lambda)=p\left(\theta_{1}, \theta_{2}\right)\left\|\begin{array}{ll}
\frac{\partial \theta_{1}}{\partial \xi^{\prime}} & \frac{\partial \theta_{1}}{\partial \lambda^{\prime}} \\
\frac{\partial \theta_{2}}{\partial \xi^{\prime}} & \frac{\partial \theta_{2}}{\partial \lambda^{\prime}}
\end{array}\right\|
$$

For evaluating the Jacobian in (16), we need the inverse of the transformation defined by $\xi=\theta_{1}$ and equation (15). The necessary expressions are given in the appendix. Now, a prior on the nonredundant EQI, consistent with the equality restrictions, can be defined as

$$
p\left(\theta_{1}\right)=\left.p(\xi \mid \lambda)\right|_{\lambda=0}=\left.\frac{\left.p(\xi, \lambda)\right|_{\lambda=0}}{\left.p(\lambda)\right|_{\lambda=0}} \propto p(\xi, \lambda)\right|_{\lambda=0}
$$

Applying this procedure to the 15-dimensional uniform prior $p(\theta)$ specified earlier, we obtain

$$
p\left(\theta_{1}\right) \propto \frac{1}{\bar{s}_{1} \bar{s}_{2}^{2}\left(1-\bar{s}_{1}-\bar{s}_{2}\right)^{3}} I_{R}(\theta)
$$

where $I_{R}(\theta)$ is an indicator function which takes the value 1 when the shares and elasticities fall within the bounds defined by the uniform priors, and 0 otherwise. Inclusion of the inequality constraints from monotonicity and concavity is deferred until after transformation to $\gamma_{A}$ and $\gamma_{B}$; imposing these constraints at each data point means they are model dependent.

Conditioning on $\lambda=0$ as a device for setting up a prior to accommodate equality restrictions on the parameters is along the lines of work by Kleibergen. However, in our specification, $\xi$ and $\lambda$ do not exhibit the orthogonality necessary to achieve invariance with respect to the conditioning vector. This fact is likely to be the 
reason that $\bar{s}_{1}, \bar{s}_{2}$ and $\bar{s}_{3}$ do not appear symmetrically in $p\left(\theta_{1}\right)$. Nevertheless, for model averaging purposes, both models are treated the same and have relatively noninformative priors.

To derive the prior pdf's for the model parameters from those for the EQI, we write, for the AIDS model,

$$
p\left(\gamma_{A}\right)=p\left(\theta_{1}\right)\left\|\frac{\partial \theta_{1}}{\partial \gamma_{A}{ }^{\prime}}\right\| I_{R^{*}}\left(\gamma_{A}\right)=\frac{k_{A} I_{R^{*}}\left(\gamma_{A}\right)}{\alpha_{A 1}^{3} \alpha_{A 2}^{3}\left(1-\alpha_{A 1}-\alpha_{A 2}\right)^{4}}
$$

where $I_{R^{*}}\left(\gamma_{A}\right)$ is an indicator function that is equal to zero if the elasticities and shares at mean prices and expenditure fall outside the regions defined by the uniform priors, or if the elasticities and shares violate monotonicity or concavity at one or more of the sample observations. The unknown normalizing constant $k_{A}$ contains components from the uniform priors, the conditioning in (17), and the truncation from imposing monotonicity and concavity. Following a similar procedure for the LTL model, we obtain

$$
p\left(\gamma_{B}\right)=p\left(\theta_{1}\right)\left\|\frac{\partial \theta_{1}}{\partial \gamma_{B}{ }^{\prime}}\right\| I_{R^{*}}\left(\gamma_{B}\right)=\frac{k_{B} I_{R^{*}}\left(\gamma_{B}\right)}{\alpha_{B 1}^{3} \alpha_{B 2}^{3}\left(1-\alpha_{B 1}-\alpha_{B 2}\right)^{4}}
$$

The expressions necessary for evaluating the Jacobians in (19) and (20) are given in the appendix. The data were scaled so that mean prices and expenditure were equal to unity, making these expressions, and others involving shares and elasticities evaluated at the means, relatively simple.

The final step for specification of prior pdf's for the parameters is the setting of priors for $\Sigma_{A}$ and $\Sigma_{B}$. These matrices are both covariance matrices for the shares 
$\left(s_{1}, s_{2}\right)$, and so it is reasonable to assign them the same prior. We chose the inverted Wishart pdf

$$
p\left(\Sigma_{B}\right)=p\left(\Sigma_{A}\right)=h\left|\Sigma_{\ell}\right|^{-(v+3) / 2} \exp \left\{-\frac{1}{2} \operatorname{tr}\left(C \Sigma_{\ell}^{-1}\right)\right\} \quad \ell=A, B
$$

with $v=2$ and $C=\operatorname{diagonal}(0.00016,0.00016)$. This prior is a proper, but relatively noninformative one. The value $v=2$ is the smallest value of $v$ for which $p\left(\Sigma_{\ell}\right)$ is proper. Setting $c_{i i}=0.00016$ implies a prior probability of 0.05 that the standard deviation of a share exceeds 0.2 . Making $C$ diagonal implies the shares are a priori independent. Such independence is unlikely, but it does let the data be the main determinant of the posterior correlation. The normalizing constant is a known value. See, for example, Zellner (p.395).

Taking $\gamma_{\ell}$ and $\Sigma_{\ell},(\ell=A, B)$, as a priori independent yields, for the joint prior pdf for the parameters of each model

$$
\begin{aligned}
p\left(\gamma_{\ell}, \Sigma_{\ell}\right) & =p\left(\gamma_{\ell}\right) p\left(\Sigma_{\ell}\right) \\
& =\frac{h k_{\ell} I_{R^{*}}\left(\gamma_{\ell}\right)}{\alpha_{\ell 1}^{3} \alpha_{\ell 2}^{3}\left(1-\alpha_{\ell 1}-\alpha_{\ell 2}\right)^{4}}\left|\Sigma_{\ell}\right|^{-(v+3) / 2} \exp \left\{-\frac{1}{2} \operatorname{tr}\left(C \Sigma_{\ell}^{-1}\right)\right\} \quad \ell=A, B
\end{aligned}
$$

\section{Posterior Results for Model Parameters}

Using Bayes' theorem to combine the prior pdf's in (22) with the likelihood functions in (11) and (12), and integrating out $\Sigma_{A}\left(\right.$ or $\left.\Sigma_{B}\right)$ yields 


$$
\begin{aligned}
p\left(\gamma_{\ell} \mid s, M_{\ell}\right) & =\int p\left(\gamma_{\ell}, \Sigma_{\ell} \mid s, M_{\ell}\right) d \Sigma_{\ell} \\
& \propto \int p\left(\gamma_{\ell}, \Sigma_{\ell}\right) p\left(s \mid \gamma_{\ell}, \Sigma_{\ell}, M_{\ell}\right) d \Sigma_{\ell} \\
& \propto \frac{I_{R^{*}}\left(\gamma_{\ell}\right)}{\alpha_{\ell 1}^{3} \alpha_{\ell 2}^{3}\left(1-\alpha_{\ell 1}-\alpha_{\ell 2}\right)^{4}} \int\left|\Sigma_{\ell}\right|^{-(T+v+3) / 2} \exp \left\{-\frac{1}{2} \operatorname{tr}\left(\left(W_{\ell}+C\right) \Sigma_{\ell}^{-1}\right)\right\} d \Sigma_{\ell} \\
& \propto \frac{I_{R^{*}}\left(\gamma_{\ell}\right)}{\alpha_{\ell 1}^{3} \alpha_{\ell 2}^{3}\left(1-\alpha_{\ell 1}-\alpha_{\ell 2}\right)^{4}}\left|W_{\ell}+C\right|^{-(v+T) / 2} \quad \ell=A, B
\end{aligned}
$$

These posterior pdf's are intractable ones; Markov chain Monte Carlo techniques are needed to estimate their moments and marginal posterior pdf's. To draw observations from (23), we used a random-walk Metropolis Hastings algorithm, with a multivariate normal transition density, with covariance matrix equal to a scalar multiplied by the maximum likelihood parameter covariance matrix. The scalar was chosen to give an acceptance rate of $40 \%-50 \%$ and a series that appeared stationary. A total of 50,000 observations were generated with the first 10,000 being discarded as a burn-in. Details of this algorithm, used in a different application, can be found in Griffiths and Chotikapanich.

Posterior means and standard deviations estimated from these draws are presented in Table 1, along with the corresponding unrestricted maximum likelihood estimates, and their standard errors. The close similarity between the two sets of estimates is perhaps surprising given the extensive inequality constraints imposed during Bayesian estimation. With the exception of the parameters $\alpha_{\ell i}, \quad(\ell=A, B$; $i=1,2$ ), that are estimated with a great deal of precision, the posterior standard deviations are large relative to the corresponding posterior means; estimation of the parameters has been relatively imprecise. Of more interest are estimates of the shares and elasticities. The draws of $\gamma_{A}$ and $\gamma_{B}$ from (23) were used to compute corresponding draws of the EQI, that can then be used to estimate the posterior 
means, standard deviations and marginal posterior pdf's of these EQI. Since we are also interested in the results from model averaging the EQI, we defer discussion of these estimates until after we have discussed the model averaging process.

\section{Model Averaging}

Having obtained the posterior pdf's for the models' parameters, the next step towards model averaging is to estimate the marginal likelihood functions that are given by

$$
\begin{aligned}
p\left(s \mid M_{\ell}\right) & =\iint p\left(s \mid \gamma_{\ell}, \Sigma_{\ell}, M_{\ell}\right) p\left(\gamma_{\ell}\right) p\left(\Sigma_{\ell}\right) d \Sigma_{\ell} d \gamma_{\ell} \\
& =\int p^{*}\left(s \mid \gamma_{\ell}, M_{\ell}\right) p\left(\gamma_{\ell}\right) d \gamma_{\ell} \quad \ell=A, B
\end{aligned}
$$

where, from (23),

$$
p^{*}\left(s \mid \gamma_{\ell}, M_{\ell}\right)=k^{*}\left|C+W_{\ell}\right|^{-(v+T) / 2}
$$

and

$$
p^{*}\left(s \mid \gamma_{\ell}, M_{\ell}\right) p\left(\gamma_{\ell}\right)=\frac{k^{*}\left|C+W_{\ell}\right|^{-(v+T) / 2} k_{\ell} I_{R^{*}}\left(\gamma_{\ell}\right)}{\alpha_{\ell 1}^{3} \alpha_{\ell 2}^{3}\left(1-\alpha_{\ell 1}-\alpha_{\ell 2}\right)^{4}}
$$

In these expressions, $p^{*}\left(s \mid \gamma_{\ell}, M_{\ell}\right)$ can be regarded as the data density or likelihood with the covariance matrix integrated out. The constant $k^{*}$ is the same for both models. Because it cancels out when computing posterior model probabilities, it can be ignored when estimating the marginal likelihoods.

The constants $k_{\ell}$ are more of a problem, however. We estimated these quantities by applying a Metropolis-Hastings algorithm to each of the priors. To see how, let $p\left(\gamma_{\ell}\right)=k_{\ell} g\left(\gamma_{\ell}\right)$. Also, let the transition density for $\gamma_{\ell}^{*}$ given $\gamma_{\ell}^{(m)}$, in a 
Metropolis-Hastings algorithm, be given by $q\left(\gamma_{\ell}^{(m)}, \gamma_{\ell}^{*}\right)$, and let the candidate draw at the $m$-th iteration be denoted by $\gamma_{\ell}^{(m)}$. Then, we can write

$$
\int p\left(\gamma_{\ell}\right) d \gamma_{\ell}=\int k_{\ell} g\left(\gamma_{\ell}\right) d \gamma_{\ell}=\int \frac{k_{\ell} g\left(\gamma_{\ell}^{*}\right)}{q\left(\gamma_{\ell}^{(m)}, \gamma_{\ell}^{*}\right)} q\left(\gamma_{\ell}^{(m)}, \gamma_{\ell}^{*}\right) d \gamma_{\ell}^{*}=1
$$

Following a suggestion by Geweke (p.44), $q\left(\gamma_{\ell}^{(m)}, \gamma_{\ell}^{*}\right)$ can be used as an importance sampling density, implying we can estimate $k_{\ell}$ as

$$
\hat{k}_{\ell}=\left(\frac{1}{M} \sum_{m=1}^{M} \frac{g\left(\gamma_{\ell}^{*(m)}\right)}{q\left(\gamma_{\ell}^{(m)}, \gamma_{\ell}^{*(m)}\right)}\right)^{-1}
$$

where $M$ is the number of post burn-in draws from the Metropolis-Hastings chain. A multivariate normal transition density, with considerable experimentation to find a suitable covariance matrix, was used with a random-walk Metropolis-Hastings chain. The estimates obtained, with numerical standard errors in parentheses, were $\log \hat{k}_{A}=15.3576(0.1428)$ and $\log \hat{k}_{B}=14.4883(0.3079)$.

It is now possible to proceed with estimation of the marginal likelihoods given in equation (24). The harmonic-mean method, suggested by Gelfand and Dey (1994), and described further by Geweke (1999, p.46) was used for this purpose. The estimate is computed from

$$
\hat{p}\left(s \mid M_{\ell}\right)=\left(\frac{1}{M} \sum_{m=1}^{M} \frac{f\left(\gamma_{\ell}^{(m)}\right)}{p^{*}\left(s \mid \gamma_{\ell}^{(m)}, M_{\ell}\right) p\left(\gamma_{\ell}^{(m)}\right)}\right)^{-1}
$$

where the $\gamma_{\ell}^{(m)}$ are the post burn-in draws from the posterior Metropolis-Hastings chain, and $f\left(\gamma_{\ell}\right)$ is the truncated normal distribution

$$
f\left(\gamma_{\ell}\right)=p^{-1}(2 \pi)^{-K / 2}\left|\hat{\Sigma}_{\gamma_{\ell}}\right|^{-1 / 2} \exp \left\{-\frac{1}{2}\left(\gamma_{\ell}-\hat{\gamma}_{\ell}\right)^{\prime} \hat{\Sigma}_{\gamma_{\ell}}^{-1}\left(\gamma_{\ell}-\hat{\gamma}_{\ell}\right)\right\} I\left(\Gamma_{\ell}\right)
$$


with $\quad \hat{\gamma}_{\ell}=\frac{1}{M} \sum_{m=1}^{M} \gamma_{\ell}^{(m)}$ and $\quad \hat{\Sigma}_{\gamma_{\ell}}=\frac{1}{M} \sum_{m=1}^{M}\left(\gamma_{\ell}^{(m)}-\hat{\gamma}_{\ell}\right)\left(\gamma_{\ell}^{(m)}-\hat{\gamma}_{\ell}\right)^{\prime}$ estimated from the posterior observations. The indicator function $I\left(\Gamma_{\ell}\right)$ equals 1 for $\gamma_{\ell}$ satisfying $\left(\gamma_{\ell}-\hat{\gamma}_{\ell}\right)^{\prime} \hat{\Sigma}_{\gamma_{\ell}}^{-1}\left(\gamma_{\ell}-\hat{\gamma}_{\ell}\right) \leq q$, where $q$ is such that $P\left(\chi_{(K)}^{2} \leq q\right)=p$, and $K=7$ is the dimension of $\gamma_{\ell}$. The marginal likelihoods were estimated for alternative values of $p$ with only minor differences for different $p$ 's. The estimates obtained for $p=0.9$ and their numerical standard errors are $\log \hat{p}\left(s \mid M_{A}\right)=334.3373(0.1431)$ and $\log \hat{p}\left(s \mid M_{B}\right)=333.4562(0.3079)$. Given equal prior probabilities are assigned to each of the models, $\left[P\left(M_{A}\right)=P\left(M_{B}\right)=0.5\right]$, estimates of the posterior model probabilities are

$$
\begin{aligned}
& \hat{P}\left(M_{A} \mid s\right)=\frac{\hat{p}\left(s \mid M_{A}\right) P\left(M_{A}\right)}{\hat{p}\left(s \mid M_{A}\right) P\left(M_{A}\right)+\hat{p}\left(s \mid M_{B}\right) P\left(M_{B}\right)}=0.7071 \\
& \hat{P}\left(M_{B} \mid s\right)=\frac{\hat{p}\left(s \mid M_{B}\right) P\left(M_{B}\right)}{\hat{p}\left(s \mid M_{A}\right) P\left(M_{A}\right)+\hat{p}\left(s \mid M_{B}\right) P\left(M_{B}\right)}=0.2929
\end{aligned}
$$

Thus, the posterior odds in favor of the AIDS model relative to the LTL function are approximately 2.4 to 1 .

Given we have observations on the shares and elasticties $\theta$, drawn from the posterior pdf's conditional on each of the models, $p\left(\theta \mid s, M_{\ell}\right), \ell=A, B$, results averaged over the two models can be obtained from

$$
E(g(\theta) \mid s)=\sum_{\ell=A, B} E\left(g(\theta) \mid s, M_{\ell}\right) P\left(M_{\ell} \mid s\right)
$$

By choosing $g(\theta)=\theta$ we can compute unconditional posterior means, $g(\theta)=\theta^{2}$ allows us to compute unconditional posterior standard deviations, and letting $g(\theta)$ be 
a series of indicator functions, equal to unity within a class and zero outside, permits construction of histograms from which unconditional posterior pdf's can be estimated.

\section{Posterior Results for Economic Quantities of Interest}

Posterior means and standard deviations for the shares and elasticities, at mean prices and expenditure, for each of the models, and from model averaging, are presented in Table 2. This table also contains the unconstrained maximum likelihood estimates from each model. We can observe the following:

1. There is little difference between the Bayesian and maximum likelihood estimates, with the exception of the price and income elasticites for poultry. Imposition of the inequality constraints has changed the price elasticity of poultry from a small insignificant positive value to a negative value, and this change appears to have impacted on other poultry estimates, such as the income elasticity, that suggests poultry is an inferior good. Apart from poultry, the posterior pdf's appear to have been dominated by the sample data, as was our original intention.

2. Despite relatively imprecise estimates of the original model parameters, many of the elastcities are estimated with reasonable precision. Poultry is again the exception in this regard.

3. The posterior means and standard deviations from the two models are virtually identical. The complete posterior pdf's, graphed in Figure 2, are also identical. At first glance this result appears to be a disappointing one for Bayesian model averaging (although reassuring for demand analysts). The average is not distinguishable from the results of either model. However, the data were scaled so that mean prices and expenditure are equal to unity; at this point the 
logarithms of prices and expenditure are zero, and both models are identical. The expressions for the elasticities involve different parameters, but they have nevertheless led to the same results.

More interesting is an examination of the results at points away from the means. For this purpose, we chose a point with relatively low prices for beef and pork, a relatively high price for poultry, and relatively low expenditure. Specifically, $p_{1}=0.8, p_{2}=0.7, p_{3}=1.2$ and $x=0.8$. Posterior means and standard deviations for the EQI at this point are given in Table 3, for each of the models, and the averaged results; the complete posterior pdf's appear in Figure 3. Given the higher posterior probability for the AIDS model the average posterior pdf's tend to mimic those for the AIDS model. However, a nonzero probability for the LTL model has a noticeable impact, particularly in the case of $s_{1}, s_{3}, \eta_{13}, \eta_{22}$ and $\eta_{33}$. In the case of $\eta_{33}$, averaging has led to a bimodal distribution. Allowing for model uncertainty clearly has an impact on our inferences.

\section{Concluding Remarks}

When a particular demand system is chosen to make inferences about quantities such as shares and elasticities at different data points, the inferences drawn are conditional on the model that is selected. Different models can lead to quite different conclusions. Choosing the best-fitting model from a number of alternative models helps reduce the chance of making mistaken inferences, but, because this strategy typically ignores discarded models, it overstates the precision with which economic quantities of interest are estimated. In this paper we have described and illustrated a Bayesian model averaging procedure that solves these problems. 
There are a number of issues that require further research. Assigning a prior to economic quantities of interest, and accommodating equality restrictions with a conditional posterior pdf, raises questions about invariance with respect to the conditioning vector. Possible refinement of this procedure needs to be investigated. The imposition of inequality restrictions introduces an unknown normalizing constant into the prior. Our strategy of using draws from the prior to estimate it is relatively inefficient. More efficient alternatives could be investigated. Finally, we only estimated two models. There are many others that one could include in a model averaging framework.

\section{Appendix}

To derive the Jacobian term in equation (16), we need to express the components of $\theta_{2}$ as functions of the components of $\xi$ and $\lambda$. The required expressions are:

$$
\begin{aligned}
& \bar{\eta}_{1 x}=\lambda_{1}-\bar{\eta}_{11}-\bar{\eta}_{12}-\bar{\eta}_{13} \\
& \bar{\eta}_{2 x}=\frac{\bar{s}_{1} \bar{\eta}_{12}+\bar{s}_{1} \bar{s}_{2} \bar{\eta}_{1 x}-\bar{s}_{1} \bar{\eta}_{21}-\lambda_{2}}{\bar{s}_{1} \bar{s}_{2}} \\
& \bar{s}_{3}=1-\bar{s}_{1}-\bar{s}_{2}-\lambda_{3} \\
& \bar{\eta}_{31}=\frac{\lambda_{4}-\bar{s}_{1}-\bar{s}_{1} \bar{\eta}_{11}-\bar{s}_{2} \bar{\eta}_{21}}{\bar{s}_{3}} \\
& \bar{\eta}_{3 x}=\frac{1-\bar{s}_{1} \bar{\eta}_{1 x}-\bar{s}_{2} \bar{\eta}_{2 x}-\lambda_{5}}{\bar{s}_{3}} \\
& \bar{\eta}_{23}=\frac{\lambda_{6}-\bar{s}_{2} \bar{s}_{3} \bar{\eta}_{2 x}+\bar{s}_{3} \bar{\eta}_{32}+\bar{s}_{2} \bar{s}_{3} \bar{\eta}_{3 x}}{\bar{s}_{2}}
\end{aligned}
$$




$$
\begin{aligned}
& \bar{\eta}_{33}=\frac{\lambda_{7}-\bar{s}_{3}-\bar{s}_{1} \bar{\eta}_{13}-\bar{s}_{2} \bar{\eta}_{23}}{\bar{s}_{3}} \\
& \bar{\eta}_{22}=\lambda_{8}-\bar{\eta}_{21}-\bar{\eta}_{23}-\bar{\eta}_{2 x}
\end{aligned}
$$

The Jacobians in equations (19) and (20) require expressions for selected shares and elasticities in terms of the model parameters. These expressions are:

For Model A:

$$
\begin{aligned}
& \bar{\eta}_{11}=-1+\frac{\gamma_{A 11}}{\alpha_{A 1}}-\beta_{A 1} \\
& \bar{\eta}_{12}=\frac{\gamma_{A 12}}{\alpha_{A 1}}-\frac{\beta_{A 1} \alpha_{A 2}}{\alpha_{A 1}} \\
& \bar{\eta}_{13}=\frac{-\gamma_{A 11}-\gamma_{A 12}}{\alpha_{A 1}}-\frac{\beta_{A 1}\left(1-\alpha_{A 1}-\alpha_{A 2}\right)}{\alpha_{A 1}} \\
& \bar{s}_{1}=\alpha_{A 1} \\
& \bar{s}_{2}=\alpha_{A 2} \\
& \bar{\eta}_{21}=\frac{\gamma_{A 12}}{\alpha_{A 2}}-\frac{\beta_{A 2} \alpha_{A 1}}{\alpha_{A 2}} \\
& \bar{\eta}_{32}=\frac{-\gamma_{A 12}-\gamma_{A 22}}{1-\alpha_{A 1}-\alpha_{A 2}}+\frac{\left(\beta_{A 1}+\beta_{A 2}\right) \alpha_{A 2}}{1-\alpha_{A 1}-\alpha_{A 2}}
\end{aligned}
$$

For model B:

$$
\begin{aligned}
& \bar{\eta}_{11}=-1+\frac{\gamma_{B 11}}{\alpha_{B 1}}-\left(\gamma_{B 11}+\gamma_{B 12}+\gamma_{B 13}\right) \\
& \bar{\eta}_{12}=\frac{\gamma_{B 12}}{\alpha_{B 1}}-\left(\gamma_{B 12}+\gamma_{B 22}+\gamma_{B 23}\right)
\end{aligned}
$$




$$
\begin{aligned}
& \bar{\eta}_{13}=\frac{\gamma_{B 13}}{\alpha_{B 1}}+\left(\gamma_{B 11}+2 \gamma_{B 12}+\gamma_{B 13}+\gamma_{B 22}+\gamma_{B 23}\right) \\
& \bar{s}_{1}=\alpha_{B 1} \\
& \bar{s}_{2}=\alpha_{B 2} \\
& \bar{\eta}_{21}=\frac{\gamma_{B 12}}{\alpha_{B 2}}-\left(\gamma_{B 11}+\gamma_{B 12}+\gamma_{B 13}\right) \\
& \bar{\eta}_{32}=\frac{\gamma_{B 23}}{1-\alpha_{B 1}-\alpha_{B 2}}-\left(\gamma_{B 12}+\gamma_{B 22}+\gamma_{B 23}\right)
\end{aligned}
$$

\section{References}

Alston, J.M., J.A. Chalfant and N.E. Piggott. "A Globally Flexible Model of the Effects of Generic Advertising of Beef and Pork on U.S. Meat Demand." Selected paper, AAEA meetings 1998, Salt Lake City, Utah.

Chalfant, J.A, R.S. Gray and K.J. White. "Evaluating Prior Beliefs in a Demand System: The Case of Meat Demand in Canada." American Journal of Agricultural Economics 73(1991):466-489.

Danilov, D.L. and J.R. Magnus. "On the Harm that Pretesting Does." CentER Discussion Paper No. 2001-37, 2001, Tilberg University.

Deaton, A. and J. Muellbauer. "An Almost Ideal Demand System." American Economic Review 70(1980):312-326.

Gelfand, A.E. and D.K. Dey. "Bayesian Model Choice: Asymptotics and Exact Calculations." Journal of the Royal Statistical Society Series B 56(1994):501514.

Geweke, J. “Using Simulation Methods For Bayesian Econometric Models: Inference, Development, and Communciation." Econometric Reviews 18(1999):1-73. 
Gordon, S. "Using Mixtures of Flexible Functional Forms to Estimate Factor Demand Elasticities." Canadian Journal of Economics 29(1996): 717-736.

Griffiths, W.E. and D. Chotikapanich. "Bayesian Methodology for Imposing Inequality Constraints on a Linear Expenditure Function with Demographic Factors." Australian Economic Papers 36(1997): 321-341.

Jorgenson, D.W., L.J. Lau and T.M. Stoker. “The Transcendental Logarithmic Model of Aggregate Consumer Behaviour,” in R.L Basmann and G. Rhodes (eds.), Advances in Econometrics (Vol 1). Greenwich: JAI Press, 1982, p.197-238.

Judge, G.G. and M.E. Bock. The Statistical Implications of Pre-Test and Stein-Rule Estimators in Econometrics. Amsterdam: North-Holland, 1978.

Judge, G.G., W.E. Griffiths, R.C. Hill, H. Lütkepohl and T.-C. Lee. The Theory and Practice of Econometrics, second edition. New York: John Wiley and Sons, 1985.

Kleibergen, F. "Equality Restricted Random Variables: Densities and Sampling Algorithms.” Erasmus University Econometric Institute Report No 9662, 1997, Rotterdam.

Moschini, G. "Imposing Local Curvature Conditions in Flexible Demand Systems." Journal of Business and Economic Statistics, 17(1999):487-490.

Piggott, N.E. The Benefits and Costs of Generic Advertising of Agricultural Commodities. PhD dissertation, University of California, Davis, 1997.

Pollak, R.A. and T.J. Wales. Demand System Specification and Estimation. Oxford: Oxford University Press, 1992.

Ryan, D.L., and T.J. Wales. "A Simple Method for Imposing Local Curvature in Some Flexible Consumer -Demand Systems." Journal of Business and Economic Statistics, 16(1998):331-338. 
Zellner, A. An Introduction to Bayesian Inference in Econometrics. New York: John Wiley and Sons, 1971. 
Figure 1. Flowchart Describing Model Averaging of Two Models.

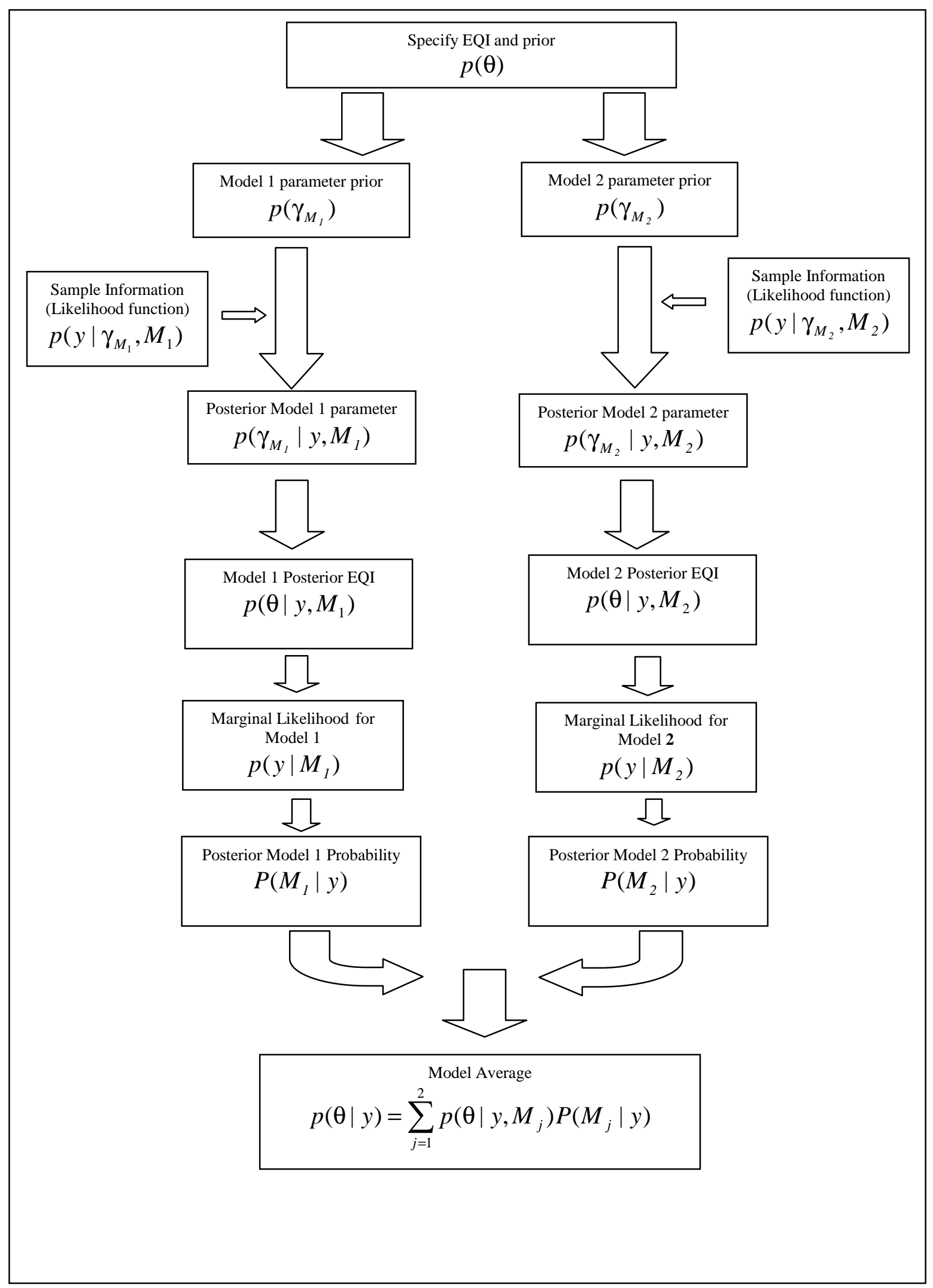


Table 1. Parameter Estimates

\begin{tabular}{|c|c|c|c|c|}
\hline \multirow{2}{*}{$\begin{array}{c}\text { Model } \\
\text { Parameters }\end{array}$} & \multicolumn{2}{|c|}{ AIDS } & \multicolumn{2}{|c|}{ LTL } \\
\hline & MLE & Bayesian & MLE & Bayesian \\
\hline$\alpha_{\ell 1}$ & $\begin{array}{c}0.5469 \\
(0.0043)\end{array}$ & $\begin{array}{c}0.5472 \\
(0.0042)\end{array}$ & $\begin{array}{c}0.5467 \\
(0.0042)\end{array}$ & $\begin{array}{c}0.5467 \\
(0.0042)\end{array}$ \\
\hline$\alpha_{\ell 2}$ & $\begin{array}{c}0.2741 \\
(0.0013)\end{array}$ & $\begin{array}{c}0.2741 \\
(0.0013)\end{array}$ & $\begin{array}{c}0.2741 \\
(0.0013)\end{array}$ & $\begin{array}{c}0.2741 \\
(0.0013)\end{array}$ \\
\hline$\beta_{A 1}$ & $\begin{array}{c}0.4001 \\
(0.1559)\end{array}$ & $\begin{array}{c}0.3253 \\
(0.1422)\end{array}$ & & \\
\hline$\beta_{A 2}$ & $\begin{array}{l}-0.0392 \\
(0.0502)\end{array}$ & $\begin{array}{l}-0.0343 \\
(0.0529)\end{array}$ & & \\
\hline$\gamma_{\ell 11}$ & $\begin{array}{c}0.0958 \\
(0.0640)\end{array}$ & $\begin{array}{c}0.0526 \\
(0.0504)\end{array}$ & $\begin{array}{c}-0.3448 \\
(0.1733)\end{array}$ & $\begin{array}{c}-0.3213 \\
(0.1659)\end{array}$ \\
\hline$\gamma_{\ell 12}$ & $\begin{array}{l}-0.0221 \\
(0.0202)\end{array}$ & $\begin{array}{c}-0.0203 \\
(0.0203)\end{array}$ & $\begin{array}{l}-0.1134 \\
(0.0394)\end{array}$ & $\begin{array}{c}-0.0926 \\
(0.0348)\end{array}$ \\
\hline$\gamma_{B 13}$ & & & $\begin{array}{c}0.0522 \\
(0.0630)\end{array}$ & $\begin{array}{c}0.0779 \\
(0.0590)\end{array}$ \\
\hline$\gamma_{\ell 22}$ & $\begin{array}{c}0.0667 \\
(0.0161)\end{array}$ & $\begin{array}{c}0.0657 \\
(0.0168)\end{array}$ & $\begin{array}{c}0.0869 \\
(0.0276)\end{array}$ & $\begin{array}{c}0.0823 \\
(0.0278)\end{array}$ \\
\hline$\gamma_{B 23}$ & & & $\begin{array}{c}0.0636 \\
(0.0465)\end{array}$ & $\begin{array}{c}0.0421 \\
(0.0405)\end{array}$ \\
\hline
\end{tabular}


Table 2. Estimates of Economic Quantities of Interest Evaluated at Means

\begin{tabular}{|c|c|c|c|c|c|}
\hline \multirow{2}{*}{ EQI } & \multicolumn{2}{|c|}{ MLE } & \multicolumn{2}{|c|}{ Bayesian } & \multirow[t]{2}{*}{ BMA } \\
\hline & AIDS & Log TL & AIDS & Log TL & \\
\hline $\bar{s}_{1}$ & $\begin{array}{c}0.5469 \\
(0.0043)\end{array}$ & $\begin{array}{c}0.5467 \\
(0.0042)\end{array}$ & $\begin{array}{c}0.5472 \\
(0.0042)\end{array}$ & $\begin{array}{c}0.5467 \\
(0.0042)\end{array}$ & $\begin{array}{c}0.5471 \\
(0.0042)\end{array}$ \\
\hline $\bar{s}_{2}$ & $\begin{array}{c}0.2741 \\
(0.0013)\end{array}$ & $\begin{array}{c}0.2741 \\
(0.0013)\end{array}$ & $\begin{array}{c}0.2741 \\
(0.0013)\end{array}$ & $\begin{array}{c}0.2741 \\
(0.0013)\end{array}$ & $\begin{array}{c}0.2741 \\
(0.0013)\end{array}$ \\
\hline $\bar{s}_{3}$ & $\begin{array}{c}0.1789 \\
(0.0036)\end{array}$ & $\begin{array}{c}0.1792 \\
(0.0035)\end{array}$ & $\begin{array}{c}0.1787 \\
(0.0035)\end{array}$ & $\begin{array}{c}0.1793 \\
(0.0035)\end{array}$ & $\begin{array}{c}0.1789 \\
(0.0035)\end{array}$ \\
\hline $\bar{\eta}_{11}$ & $\begin{array}{l}-1.2250 \\
(0.1745)\end{array}$ & $\begin{array}{l}-1.2248 \\
(0.1786)\end{array}$ & $\begin{array}{c}-1.2291 \\
(0.1729)\end{array}$ & $\begin{array}{l}-1.2519 \\
(0.1738)\end{array}$ & $\begin{array}{c}-1.2358 \\
(0.1735)\end{array}$ \\
\hline $\bar{\eta}_{22}$ & $\begin{array}{c}-0.7177 \\
(0.0668)\end{array}$ & $\begin{array}{l}-0.7202 \\
(0.0641)\end{array}$ & $\begin{array}{c}-0.7260 \\
(0.0660)\end{array}$ & $\begin{array}{l}-0.7317 \\
(0.0653)\end{array}$ & $\begin{array}{c}-0.7277 \\
(0.0659)\end{array}$ \\
\hline $\bar{\eta}_{33}$ & $\begin{array}{c}0.0212 \\
(0.3203)\end{array}$ & $\begin{array}{c}0.0434 \\
(0.3297)\end{array}$ & $\begin{array}{c}-0.2744 \\
(0.1993)\end{array}$ & $\begin{array}{c}-0.2771 \\
(0.1900)\end{array}$ & $\begin{array}{c}-0.2752 \\
(0.1966)\end{array}$ \\
\hline $\bar{\eta}_{12}$ & $\begin{array}{l}-0.2410 \\
(0.0884)\end{array}$ & $\begin{array}{l}-0.2444 \\
(0.0903)\end{array}$ & $\begin{array}{c}-0.2001 \\
(0.0787)\end{array}$ & $\begin{array}{l}-0.2012 \\
(0.0783)\end{array}$ & $\begin{array}{l}-0.2004 \\
(0.0786)\end{array}$ \\
\hline $\bar{\eta}_{13}$ & $\begin{array}{c}-0.2655 \\
(0.1068)\end{array}$ & $\begin{array}{l}-0.2733 \\
(0.1226)\end{array}$ & $\begin{array}{l}-0.1654 \\
(0.0821)\end{array}$ & $\begin{array}{l}-0.1617 \\
(0.0788)\end{array}$ & $\begin{array}{l}-0.1643 \\
(0.0812)\end{array}$ \\
\hline $\bar{\eta}_{21}$ & $\begin{array}{l}-0.0026 \\
(0.1172)\end{array}$ & $\begin{array}{l}-0.0076 \\
(0.1183)\end{array}$ & $\begin{array}{c}-0.0058 \\
(0.1258)\end{array}$ & $\begin{array}{l}-0.0019 \\
(0.1237)\end{array}$ & $\begin{array}{l}-0.0046 \\
(0.1252)\end{array}$ \\
\hline $\bar{\eta}_{23}$ & $\begin{array}{l}-0.1368 \\
(0.0847)\end{array}$ & $\begin{array}{l}-0.1370 \\
(0.0863)\end{array}$ & $\begin{array}{c}-0.1432 \\
(0.0886)\end{array}$ & $\begin{array}{c}-0.1506 \\
(0.0853)\end{array}$ & $\begin{array}{l}-0.1454 \\
(0.0877)\end{array}$ \\
\hline $\bar{\eta}_{31}$ & $\begin{array}{c}0.6917 \\
(0.4365)\end{array}$ & $\begin{array}{c}0.6974 \\
(0.4473)\end{array}$ & $\begin{array}{c}0.7097 \\
(0.4265)\end{array}$ & $\begin{array}{c}0.7707 \\
(0.4321)\end{array}$ & $\begin{array}{c}0.7275 \\
(0.4291)\end{array}$ \\
\hline $\bar{\eta}_{32}$ & $\begin{array}{c}0.3041 \\
(0.2232)\end{array}$ & $\begin{array}{c}0.3176 \\
(0.2303)\end{array}$ & $\begin{array}{c}0.1919 \\
(0.2024)\end{array}$ & $\begin{array}{c}0.2029 \\
(0.1980)\end{array}$ & $\begin{array}{r}0.1951 \\
(0.2012)\end{array}$ \\
\hline $\bar{\eta}_{1 x}$ & $\begin{array}{c}1.7315 \\
(0.2854)\end{array}$ & $\begin{array}{c}1.7426 \\
(0.2939)\end{array}$ & $\begin{array}{c}1.5946 \\
(0.2603)\end{array}$ & $\begin{array}{c}1.6148 \\
(0.2593)\end{array}$ & $\begin{array}{r}1.6005 \\
(0.2602)\end{array}$ \\
\hline $\bar{\eta}_{2 x}$ & $\begin{array}{c}0.8570 \\
(0.1830)\end{array}$ & $\begin{array}{c}0.8648 \\
(0.1438)\end{array}$ & $\begin{array}{c}0.8750 \\
(0.1929)\end{array}$ & $\begin{array}{c}0.8841 \\
(0.1869)\end{array}$ & $\begin{array}{c}0.8777 \\
(0.1912)\end{array}$ \\
\hline $\bar{\eta}_{3 x}$ & $\begin{array}{l}-1.0169 \\
(0.7292)\end{array}$ & $\begin{array}{l}-1.0584 \\
(0.7488)\end{array}$ & $\begin{array}{c}-0.6271 \\
(0.6456)\end{array}$ & $\begin{array}{l}-0.6965 \\
(0.6504)\end{array}$ & $\begin{array}{l}-0.6475 \\
(0.6478)\end{array}$ \\
\hline
\end{tabular}


Table 3. Estimates of Economic Quantities of Interest Evaluated away from the Means.

\begin{tabular}{|c|c|c|c|}
\hline \multirow{2}{*}{ EQI } & \multicolumn{2}{|c|}{ Bayesian } & BMA \\
\hline & AIDS & $\log T L$ & \\
\hline$s_{1}$ & $\begin{array}{c}0.5221 \\
(0.0168)\end{array}$ & $\begin{array}{c}0.5278 \\
(0.0139)\end{array}$ & $\begin{array}{c}0.5238 \\
(0.0162)\end{array}$ \\
\hline$s_{2}$ & $\begin{array}{c}0.2484 \\
(0.0101)\end{array}$ & $\begin{array}{c}0.2504 \\
(0.0085)\end{array}$ & $\begin{array}{c}0.2490 \\
(0.0097)\end{array}$ \\
\hline$S_{3}$ & $\begin{array}{c}0.2295 \\
(0.0146)\end{array}$ & $\begin{array}{c}0.2218 \\
(0.0117)\end{array}$ & $\begin{array}{c}0.2272 \\
(0.0142)\end{array}$ \\
\hline$\eta_{11}$ & $\begin{array}{l}-1.2332 \\
(0.1821)\end{array}$ & $\begin{array}{l}-1.2390 \\
(0.1549)\end{array}$ & $\begin{array}{l}-1.2349 \\
(0.1746)\end{array}$ \\
\hline$\eta_{22}$ & $\begin{array}{l}-0.6999 \\
(0.0724)\end{array}$ & $\begin{array}{l}-0.7353 \\
(0.0664)\end{array}$ & $\begin{array}{l}-0.7103 \\
(0.0725)\end{array}$ \\
\hline$\eta_{33}$ & $\begin{array}{l}-0.3959 \\
(0.1507)\end{array}$ & $\begin{array}{l}-0.5364 \\
(0.0970)\end{array}$ & $\begin{array}{l}-0.4371 \\
(0.1513)\end{array}$ \\
\hline$\eta_{12}$ & $\begin{array}{l}-0.1944 \\
(0.0806)\end{array}$ & $\begin{array}{c}-0.1837 \\
(0.0678)\end{array}$ & $\begin{array}{c}-0.1912 \\
(0.0772)\end{array}$ \\
\hline$\eta_{13}$ & $\begin{array}{l}-0.1993 \\
(0.0998)\end{array}$ & $\begin{array}{c}-0.1403 \\
(0.0668)\end{array}$ & $\begin{array}{c}-0.1820 \\
(0.0952)\end{array}$ \\
\hline$\eta_{21}$ & $\begin{array}{l}-0.0094 \\
(0.1375)\end{array}$ & $\begin{array}{c}-0.0308 \\
(0.1178)\end{array}$ & $\begin{array}{l}-0.1567 \\
(0.1324)\end{array}$ \\
\hline$\eta_{23}$ & $\begin{array}{l}-0.1576 \\
(0.1094)\end{array}$ & $\begin{array}{c}-0.1250 \\
(0.0815)\end{array}$ & $\begin{array}{l}-0.1480 \\
(0.1031)\end{array}$ \\
\hline$\eta_{31}$ & $\begin{array}{c}0.5443 \\
(0.3494)\end{array}$ & $\begin{array}{c}0.6079 \\
(0.3175)\end{array}$ & $\begin{array}{c}0.5630 \\
(0.3416)\end{array}$ \\
\hline$\eta_{32}$ & $\begin{array}{c}0.1135 \\
(0.1540)\end{array}$ & $\begin{array}{c}0.1366 \\
(0.1379)\end{array}$ & $\begin{array}{c}0.1202 \\
(0.1499)\end{array}$ \\
\hline$\eta_{1 x}$ & $\begin{array}{c}1.6269 \\
(0.2790)\end{array}$ & $\begin{array}{c}1.5630 \\
(0.2249)\end{array}$ & $\begin{array}{c}1.6082 \\
(0.2659)\end{array}$ \\
\hline$\eta_{2 x}$ & $\begin{array}{c}0.8669 \\
(0.2099)\end{array}$ & $\begin{array}{c}0.8910 \\
(0.1805)\end{array}$ & $\begin{array}{c}0.8740 \\
(0.2020)\end{array}$ \\
\hline$\eta_{3 x}$ & $\begin{array}{c}-0.2619 \\
(0.4928)\end{array}$ & $\begin{array}{c}-0.2081 \\
(0.4262)\end{array}$ & $\begin{array}{l}-0.2461 \\
(0.4749)\end{array}$ \\
\hline
\end{tabular}

1=beef; 2=pork; 3=poultry. 
Figure 2. Posterior Pdf's for Economic Quantities of Interest Evaluated at the Means
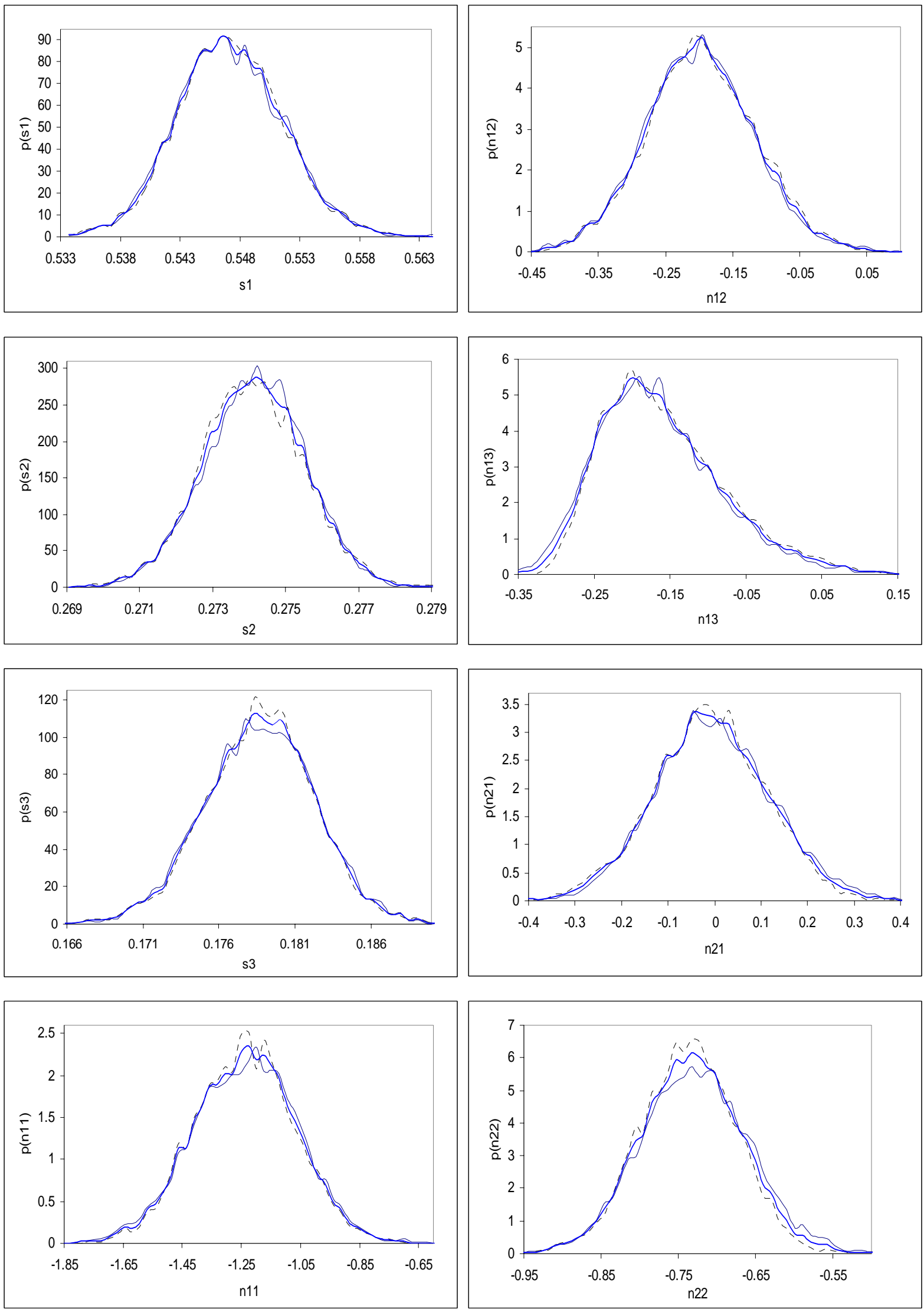

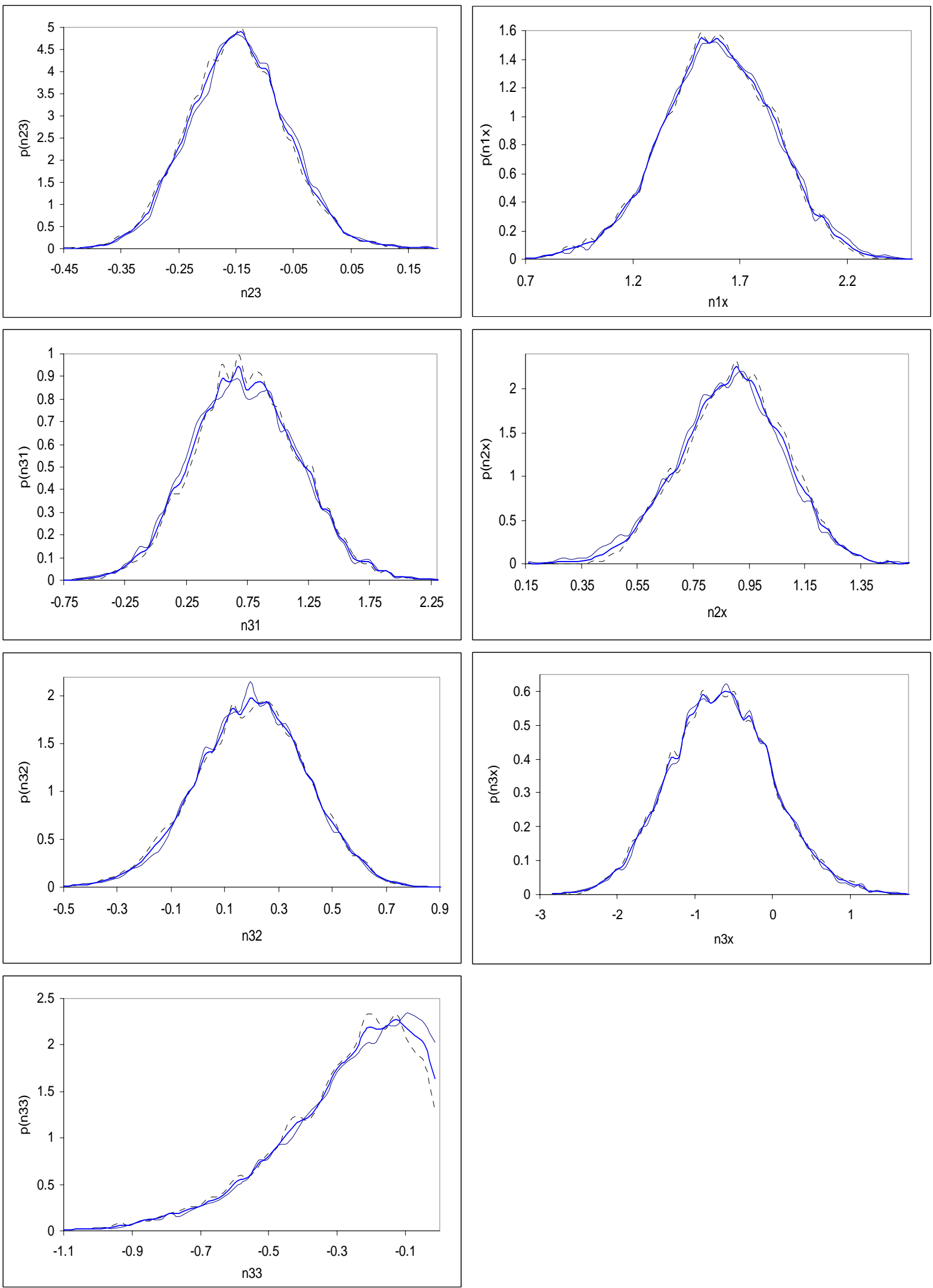

AIDS

LTL

BMA 
Figure 3. Posterior Pdf's for Economic Quantities of Interest Evaluated away from the Means
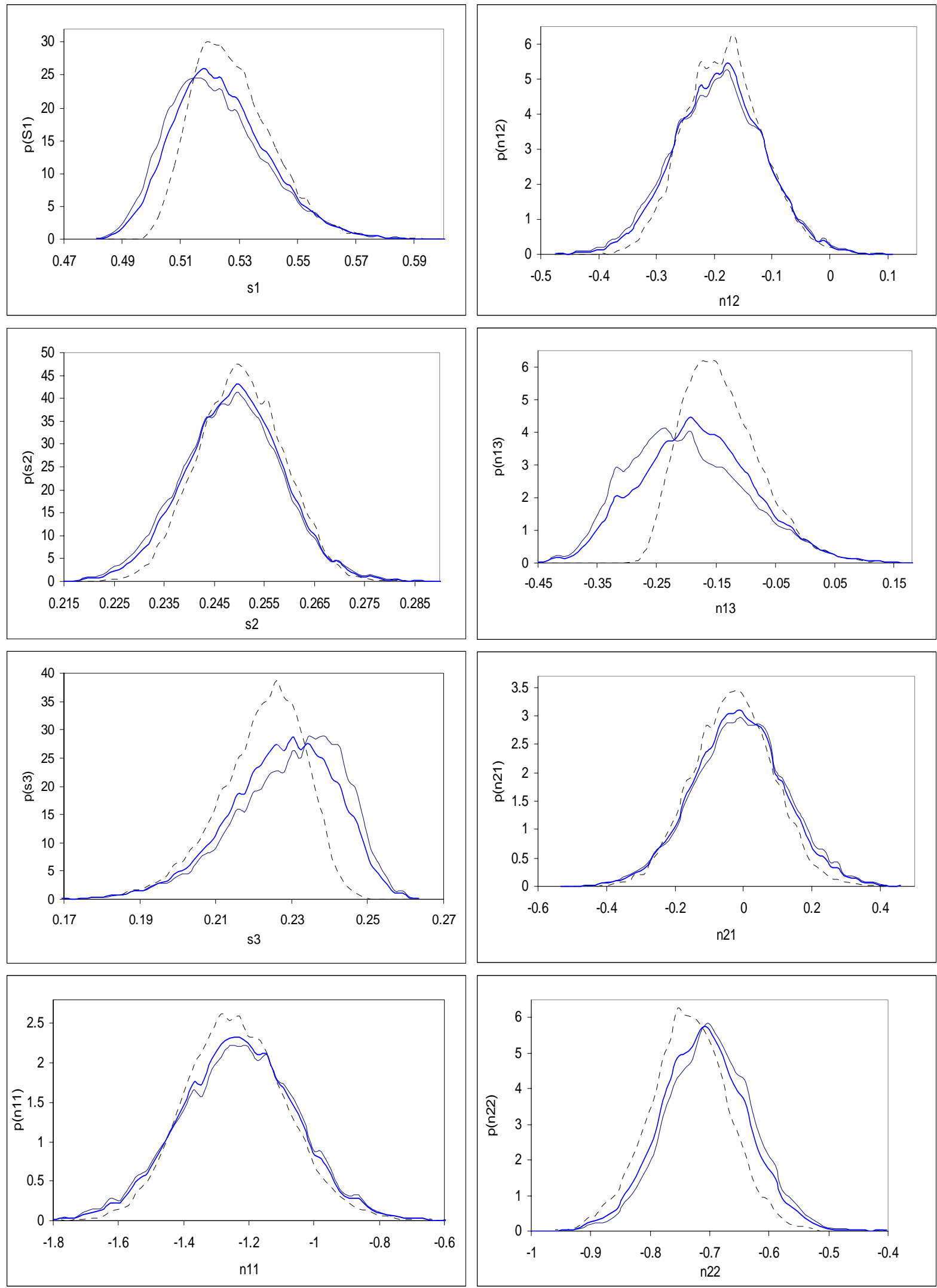

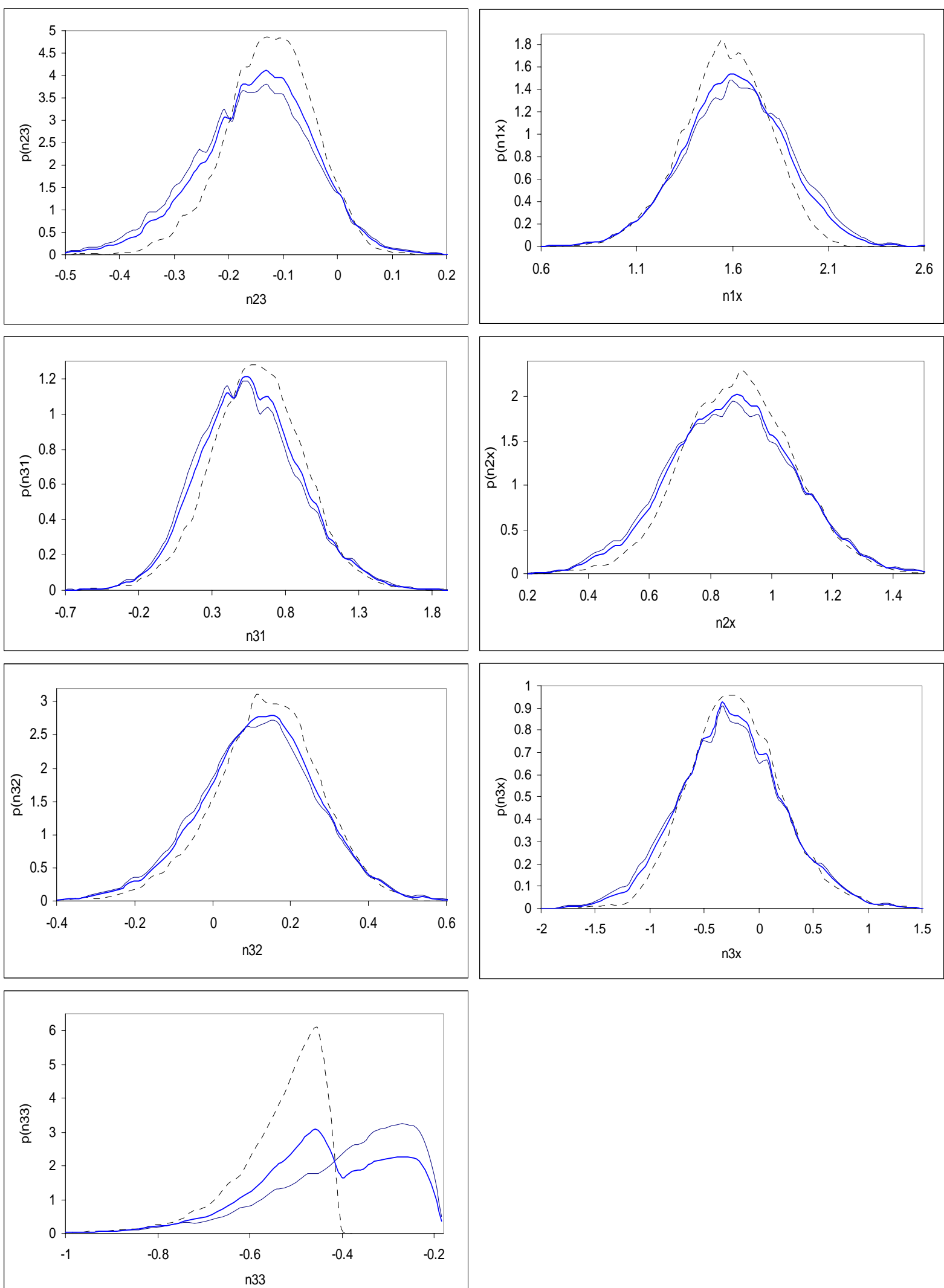

AIDS

LTL

BMA 